which may suggest shared predisposition to these autoimmune diseases and lung cancer. Advice on smoking control would be appropriate for patients with these diseases.

In conclusion, the increased risk of COPD but not lung cancer in patients with many autoimmune diseases suggests that the defined autoimmunity may predispose to COPD. Another possibility is that smoking is a risk factor for some of these autoimmune diseases and the effects on lung cancer cannot be recognised because of the small number of cases. This possibility could not be ruled out because data on smoking were lacking. The four-fold risks of lung cancer in patients with systemic sclerosis, discoid lupus erythematosus and polymyositis/dermatomyositis may indicate susceptibility to lung cancer, which should be considered in the counselling of these patients.

\section{K. Hemminki*,\# ${ }^{*}$ X. Liü ${ }^{\#}$ J. Ji ${ }^{\#}$, K. Sundquist ${ }^{\#}$ and J. Sundquist ${ }^{\#, \text { 厂 }}$}

*Division of Molecular Genetic Epidemiology, German Cancer Research Center (DKFZ), Heidelberg, Germany, "Center for Health Care Research, Lund University, Malmö, Sweden, and 'Stanford Prevention Research Center, Stanford University School of Medicine, Stanford, CA, USA.

Correspondence: K. Hemminki, DKFZ, Im Neuenheimer Feld 580, D-69120 Heidelberg, Germany. E-mail: k.hemminki @dkfz.de

Support Statement: Supported by the Swedish Council for Working Life and Social Research (Stockholm, Sweden), the Swedish Cancer Society (Stockholm, Sweden) and Deutsche Krebshilfe (Bonn, Germany).
Acknowledgements: The used database was created by linking registers maintained at Statistics Sweden (Stockholm, Sweden) and the Swedish Cancer Registry.

\section{REFERENCES}

1 Zhernakova A, van Diemen CC, Wijmenga C. Detecting shared pathogenesis from the shared genetics of immune-related diseases. Nat Rev Genet 2009; 10: 43-55.

2 Forabosco P, Bouzigon E, Ng MY, et al. Meta-analysis of genomewide linkage studies across autoimmune diseases. Eur J Hum Genet 2009; 17: 236-243.

3 Costenbader $\mathrm{KH}$, Karlson EW. Cigarette smoking and autoimmune disease: what can we learn from epidemiology? Lupus 2006; 15: 737-745.

4 Cosio MG, Saetta M, Agusti A. Immunologic aspects of chronic obstructive pulmonary disease. N Engl J Med 2009; 360: 2445-2454.

5 Walter R, Gottlieb DJ, O'Connor GT. Environmental and genetic risk factors and gene-environment interactions in the pathogenesis of chronic obstructive lung disease. Environ Health Perspect 2000; 108: Suppl. 4, 733-742.

6 Jyrki-Tapani K, Sovijarvi A, Lundback B. Chronic obstructive pulmonary disease in Finland: prevalence and risk factors. COPD 2005; 2: 331-339.

7 Hemminki K, Li X, Sundquist K, et al. Familial risks for chronic obstructive pulmonary disease among siblings based on hospitalizations in Sweden. J Epidemiol Community Health 2008; 62: 398-401.

8 Hemminki K, Li X, Sundquist J, et al. Familial associations of rheumatoid arthritis with autoimmune disorders and related conditions. Arthritis Rheum 2009; 60: 661-668.

9 Hemminki K, Li X, Sundquist K, et al. Familial risks for asthma among twins and other siblings based on hospitalizations in Sweden. Clin Exp Allergy 2007; 37: 1320-1325.

10 Cimmino MA, Zaccaria A. Epidemiology of polymyalgia rheumatica. Clin Exp Rheumatol 2000; 18: S9-S11.

\title{
Clinical features of secondary pulmonary alveolar proteinosis: pre-mortem cases in Japan
}

\section{To the Editors:}

Pulmonary alveolar proteinosis (PAP) is a rare syndrome that predominantly affects the lungs, and is characterised by the accumulation of surfactant lipids and proteins in the alveoli and terminal airways [1]. In 1999, we published findings that high levels of granulocyte-macrophage colony-stimulating factor (GM-CSF) neutralising autoantibodies were detected specifically in patients with idiopathic PAP [2]. Recently, as a result of the excellent sensitivity and specificity of GM-CSF autoantibody assays in identifying this form of PAP [3], it has been proposed that the nomenclature for this condition should be changed to "autoimmune PAP" rather than "idiopathic $\mathrm{PAP}^{\prime \prime}$ [4]. Secondary or hereditary PAP is not associated with
GM-CSF autoantibodies but develops as a consequence of a separate underlying disorder or genetic background [5]. Recently, we demonstrated that the characteristic high-resolution computed tomography (HRCT) findings in secondary PAP are distinct from those in autoimmune PAP [6]. However, physicians may not suspect secondary PAP, even when encountering unexplained pulmonary opacities on chest radiograph or computed tomography in patients with pre-existing haematological or infectious diseases, since there is little information available on the clinical features. Here, we outline the clinical features of adult-onset secondary PAP based on our database of 40 pre-mortem cases in which serum GM-CSF autoantibodies were negative, and compare them with other cases in the literature. 
A total of 404 patients with pre-mortem cytologically or pathologically proven PAP were registered in our study group between 1999 and 2009. We obtained consent from all treating physicians for each identified case according to the guidelines for epidemiological studies from the Ministry of Health, Labour and Welfare [7], and all clinical data were deidentified. $360(89 \%)$ cases were positive for serum GM-CSF autoantibody. The clinical features of 223 of the cases were previously published as a cross-sectional study [4]. 40 (10\%) cases were negative for serum GM-CSF autoantibody, and were diagnosed as secondary PAP based on the existence of appropriate underlying diseases (table 1). Four (1\%) cases remained as unclassified PAP.

Definitive diagnosis of secondary PAP was made using bronchoalveolar lavage (BAL) in 21 cases, transbronchial lung biopsy in nine cases, and video-assisted thoracoscopic surgery in 10 cases. All cases were diagnosed based on the pathological examination of lung biopsy specimens or BAL fluid cytology. At diagnosis, underlying diseases had been recognised in 30 cases; in the other 10, underlying disease was diagnosed at the same time as secondary PAP. The median (range) age at diagnosis was 49 (24-77) yrs and male sex was dominant (1.4:1). At diagnosis, $77 \%$ of the cases were symptomatic, with the most common symptom being dyspnoea on effort $(40 \%)$ followed by fever (38\%).

Haematological disorders constituted $88 \%$ of all underlying diseases. Among these, myelodysplastic syndrome (MDS) was the most frequent (65\%). Interestingly, there were four cases of Behcet's disease complicated by MDS (two of which developed MDS during the treatment of intestinal Behcet's disease) and a single case of Behcet's disease alone [8]. The other non-haematological underlying diseases consisted of single cases of Wegener's granulomatosis, pulmonary tuberculosis, nontuberculosis mycobacterium infection, and microscopic polyangitis.

At diagnosis, arterial blood gas analysis was performed in 29 cases and the mean \pm SD alveolar-arterial oxygen difference was $46.8 \pm 55.6 \mathrm{mmHg}$. HRCT was performed in 27 cases, with the findings contributing significantly to a definitive diagnosis. In the present study, six new cases were added to the 21 cases from our previous study [6], and we reanalysed HRCT characteristics in all 27 cases. In accordance with our previous report, HRCT findings were classified into three patterns based on the nature of the observed ground-glass opacities. 17 (63\%) cases showed a diffuse pattern in which the ground-glass opacities were evenly distributed with indistinct borders. In five $(19 \%)$ cases, a patchy geographic pattern was seen, and a mixed pattern was seen in the remaining five (19\%) cases. The so-called "crazy-paving" appearance was rare in secondary PAP (12\%).

25 cases underwent pulmonary function tests at diagnosis of PAP. As is the case with autoimmune PAP, forced vital capacity and forced expiratory volume in $1 \mathrm{~s}$ were within normal limits, whereas the mean $\pm \mathrm{SD}$ diffusing capacity for carbon monoxide was markedly reduced to $46.2 \pm 13.8 \%$ of normal. Serum levels of KL-6, surfactant protein (SP)-D, SP-A, and carcinoembryonic antigen were all elevated in a similar way to autoimmune PAP [4]. Among them, KL-6 was most

\begin{tabular}{|c|c|c|c|c|}
\hline TABLE 1 & \multicolumn{4}{|c|}{$\begin{array}{l}\text { Demographic characteristics and clinical } \\
\text { features of our cases and cases from the } \\
\text { literature }^{\#}\end{array}$} \\
\hline \multirow{2}{*}{\multicolumn{2}{|c|}{ Characteristics }} & \multicolumn{2}{|c|}{ Our cases } & \multirow{2}{*}{$\begin{array}{c}\text { Literature cases } \\
\text { SPAP }\end{array}$} \\
\hline & & APAP & SPAP & \\
\hline Subjects $n$ & & 223 & 40 & 57 \\
\hline Age yrs & & 50 (9-89) & 49 (24-77) & $40(24-74)$ \\
\hline \multicolumn{5}{|l|}{ Sex $n$} \\
\hline Males & & 151 & 23 & 31 \\
\hline Females & & 72 & 17 & 26 \\
\hline \multicolumn{5}{|c|}{ Clinical symptoms } \\
\hline Asymptom & & $70(32)$ & $9(23)$ & $0(0)$ \\
\hline Symptoma & & $150(68)$ & $31(77)$ & $21(100)$ \\
\hline Dyspnoe & on effort & $116(53)$ & $16(40)$ & $10(48)$ \\
\hline Febrile & & $2(1)$ & $15(38)$ & $14(67)$ \\
\hline \multicolumn{5}{|c|}{ Smoking habits } \\
\hline Never-sr & oker & $93(43)$ & $13(37)$ & NA \\
\hline Current & ex-smoker & $124(57)$ & $22(63)$ & NA \\
\hline \multicolumn{5}{|c|}{ Underlying diseases } \\
\hline Haematolo & ical disorders & None & $35(88)$ & $43(76)$ \\
\hline Myelody & lastic syndrome & & 26 & 6 \\
\hline Chronic & yyeloid leukaemia & & 2 & 16 \\
\hline Myelofib & & & 2 & 1 \\
\hline Acute $\mathrm{m}$ & loid leukaemia & & 1 & 8 \\
\hline Acute ly & phoid leukaemia & & 1 & 3 \\
\hline Adult T-C & Il leukaemia & & 1 & 0 \\
\hline Aplastic & naemia & & 1 & 1 \\
\hline Lympho & & & 1 & 3 \\
\hline Multiple & yyeloma & & 0 & 1 \\
\hline Plasmac & oma & & 0 & 1 \\
\hline Alympho & lasia & & 0 & 1 \\
\hline Essentia & hrombocytosis & & 0 & 1 \\
\hline Infectious & seases & None & 2 (5) & $3(5)$ \\
\hline Autoimmur & diseases & None & $3(7)$ & $4(7)$ \\
\hline Post orgar & ransplantation & None & 0 & $4(7)$ \\
\hline Non-haem & ological malignancy & None & 0 & $3(5)$ \\
\hline \multicolumn{5}{|c|}{ Diagnostic procedure } \\
\hline Bronchosc & & 208 (93) & $30(75)$ & $27(47)$ \\
\hline Video-assi & ed thoracoscopy & $15(7)$ & $10(25)$ & $9(16)$ \\
\hline Open lung & iopsy & $0(0)$ & $0(0)$ & $21(37)$ \\
\hline
\end{tabular}

Data are presented as median (range) or $n(\%)$, unless otherwise stated. APAP: autoimmune pulmonary alveolar proteinosis; SPAP: secondary pulmonary alveolar proteinosis; NA: not applicable. ${ }^{\#}$ : cases from the literature were identified using the search methods detailed in the main text.

strikingly elevated in both autoimmune and secondary PAP, but not specifically so, as it is known to be elevated in a number of other lung diseases [9].

A total of 35 cases with PAP secondary to haematological disorders were followed-up for 2 yrs after diagnosis. A survival rate of $46 \%$ was noted in this group. The median survival time for all cases was 16 months; 17 of the cases died within 2 yrs of the diagnosis of secondary PAP. In one case, PAP improved after cord blood transplantation. Whole lung 
lavage was carried out in five cases, but three patients died within 2 months of the procedure (PAP was exacerbated in all three subjects with apparent progression of underlying disease in one of them). In one case, PAP was improved by bone marrow transplantation after whole lung lavage. Of the eight cases that underwent segmental BAL for treatment of PAP, four died within 6 months. In two cases, secondary PAP remained stable more than 5 yrs after diagnosis. Of the five cases with non-hematological disorders, only one patient with Behcet's disease died (13 months after diagnosis) due to respiratory failure caused by PAP.

Cases in the literatures were identified by searching Index Medicus for entries listed under "Lung Diseases" between 1958 and 1966, and by searching Medline for entries listed under "alveolar proteinosis", "alveolar lipoproteinosis", "pulmonary alveolar proteinosis" and "pulmonary alveolar lipoproteinosis" between 1966 and 2009. Proceedings of conferences and cases published only in abstract form were not systematically sought and were only identified if cited by other publications. The above search strategy identified 556 articles written in English on pulmonary alveolar proteinosis. Of those, we carefully selected 69 reports in which 139 cases with underlying diseases or states were described as the secondary development of pulmonary alveolar proteinosis. For the purposes of comparison with the present data, we excluded: 66 cases diagnosed post mortem; 10 cases in which the patient was $<20 \mathrm{yrs}$ old at diagnosis because our database does not include paediatric cases; and five cases that were also included in our present database. One case associated with dust inhalation was also excluded, given that our previous study demonstrated that this might in fact be due to autoimmune PAP [4]. Consequently, 57 cases were compiled into a database for meta-analysis (table 1). At 40 yrs of age, the median age at diagnosis was younger than in our sample. The male to female ratio was similar to ours, at 1.2:1. Consistent with our data, haematological disorders were the underlying disease in $75 \%$ of cases. Unlike our cohort, PAP secondary to MDS was only seen in six $(11 \%)$ cases, all of which were been reported after 2001 [10-12]. When we compared the clinical features of those who have secondary PAP from our study and from the literature, with those of the 223 cases with autoimmune PAP (table 1), we found that the age at diagnosis was older and the male to female ratio was higher in autoimmune PAP. In autoimmune PAP, age at diagnosis was older, and the male to female ratio was higher than those in with secondary PAP, but smoking habits were not distinctive. The proportion of symptomatic patients in autoimmune PAP was not different from those in both our cases and the cases from the literature, but febrile cases were rare. The median survival time after diagnosis in the cases from the literature $(n=44)$ was 14 months, which was not significantly different from our data. In figure 1 , the cumulative survival curve after diagnosis for our cases and for the cases from the literature demonstrates the consistently poor prognosis of secondary PAP.

Taken together, we conclude that haematological disorders are the major underlying disease in $>75 \%$ of cases with adultonset secondary PAP. The median age at diagnosis is typically in the fourth decade of life, with a greater incidence among males (1.2-1.4:1). Prognosis is very poor, with a median survival time of $<20$ months.

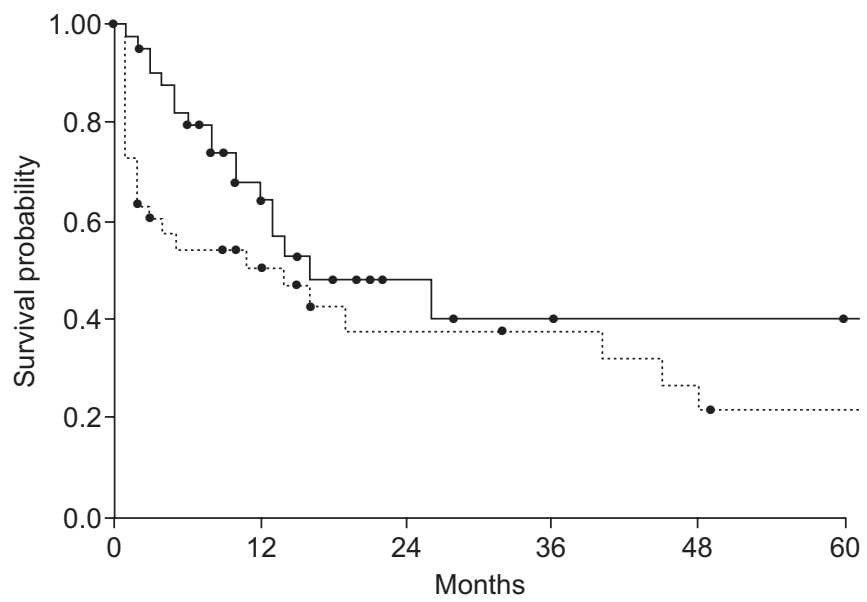

FIGURE 1. Overall survival probability of cases from our study ( - ) and cases from the literature $(\cdots \cdots \cdots \cdot . \cdot)$. The longest survival term from diagnosis of pulmonary alveolar proteinosis (PAP) was investigated in each case and data were analysed using SPSS 17.0 (SPSS, Chicago, IL, USA). In our original 40 cases, we could follow-up all cases. The longest terms of patient survival after diagnosis were confirmed in only 44 cases described in the literature.

This is the first report outlining the clinical features of secondary PAP. We believe that this information will contribute to the diagnosis and management of patients with secondary PAP.

H. Ishii*, R. Tazawa", C. Kaneko", T. Saraya*, Y. Inoue ${ }^{\natural}$, E. Hamano $^{+}$, Y. Kogure ${ }^{\S}$, K. Tomii ${ }^{f}$, M. Terada**, T. Takada**, M. Hojo"\#\#, A. Nishida ${ }^{\text {थฯ }}$, T. Ichiwata ${ }^{++}$, B.C. Trapnell ${ }^{\S \S}$, H. Goto* $^{*}$ and K. Nakata ${ }^{\#}$

*Dept of Respiratory Medicine, Kyorin University Hospital, ${ }^{+}$Dept of Pulmonary Medicine, University of Tokyo, ${ }^{\# \#}$ Dept of Respiratory Medicine, International Medical Center of Japan, " Dept of Hematology, Toranomon Hospital, ${ }^{++}$Dept of Respiratory Medicine, Tokyo Medical University Hachioji Medical Center, Tokyo, "Bioscience Medical Research Center, **Dept of Respiratory Medicine, Niigata University Medical and Dental Hospital, Niigata, "Dept of Diffuse Lung Diseases and Respiratory Failure, Clinical Research Center, NHO KinkiChuo Chest Medical Center, Osaka, ${ }^{\S}$ Dept of Respiratory Medicine, NHO Nagoya Medical Center, Aichi, ${ }^{f}$ Dept of Respiratory Medicine, Kobe City Medical Center General Hospital, Hyogo, Japan, and ${ }^{\S \S}$ Division of Pulmonary Biology and Medicine, Children's Hospital Research Foundation, Cincinnati, $\mathrm{OH}$, USA.

Correspondence: K. Nakata, Bioscience Medical Research Center, Niigata University Medical and Dental Hospital, 1-754 Asahimachi-Dohri, Chuoku, Niigata City, Niigata 9518520, Japan. E-mail: radical@med.niigata-u.ac.jp

Support Statement: The present study was in part supported by grant Category B18406031 and B21406025 from the Japan Society for the Promotion of Science, and grant H21-NanchiIppan-161 and H14-trans-014 from the Ministry of Health, 
Labor, and Welfare of Japan, as well as grants from the Ministry of Education, Culture, Sports, Science, and Technology of Japan.

Statement of Interest: None declared.

\section{REFERENCES}

1 Rosen SH, Castleman B, Liebow AA. Pulmonary alveolar proteinosis. N Engl J Med 1958; 258: 123-1143.

2 Kitamura T, Tanaka N, Watanabe J, et al. Idiopathic pulmonary alveolar proteinosis as an autoimmune disease with neutralizing antibody against granulocyte-macrophage colony stimulating factor. J Exp Med 1999; 190: 875-880.

3 Kitamura T, Uchida K, Tanaka N, et al. Serological diagnosis of idiopathic pulmonary alveolar proteinosis. Am J Respir Crit Care Med 2000; 162: 658-662.

4 Inoue $\mathrm{Y}$, Trapnell BC, Tazawa R, et al. Characteristics of a large cohort of autoimmune pulmonary alveolar proteinosis patients in Japan. Am J Respir Crit Care Med 2008; 177: 752-762.

5 Suzuki T, Sakagami T, Young LR, et al. Hereditary pulmonary alveolar proteinosis: pathogenesis, presentation, diagnosis, and therapy. Am J Respir Crit Care Med 2010; 182: 1292-1304.
6 Ishii H, Trapnell BC, Tazawa R, et al. Comparative study of highresolution CT findings between autoimmune and secondary pulmonary alveolar proteinosis. Chest 2009; 136: 1348-1355.

7 Ministry of Health, Labour and Welfare. Protecting personal identifiable data: ethical guidelines for epidemiological research Tokyo, Ministry of Health, Labour and Welfare of Japan, 2002; pp. 18-20.

8 Uchiyama M, Nagao T, Hattori A, et al. Pulmonary alveolar proteinosis in a patient with Behcet's disease. Respirology 2009; 14: 305-308.

9 Lederer DJ, Jelic S, Basner RC, et al. Circulating KL-6, a biomarker of lung injury, in obstructive sleep apnoea. Eur Respir J 2009; 33: 793-796.

10 Ohnishi T, Yamada G, Shijubo N, et al. Secondary pulmonary alveolar proteinosis associated with myelodysplastic syndrome. Intern Med 2003; 42: 187-190.

11 Pollack SM, Gutierrez G, Ascensao J. Pulmonary alveolar proteinosis with myeloproliferative syndrome with myelodysplasia: bronchoalveolar lavage reduces white blood cell count. Am J Hematol 2006; 81: 634-638.

12 Chung JH, Pipavath SJ, Myerson DH, et al. Secondary pulmonary alveolar proteinosis: a confusing and potentially serious complication of hematologic malignancy. J Thorac imaging 2009; 24: 115-118.

DOI: $10.1183 / 09031936.00092910$

\section{Is NMR-based metabolomic analysis of exhaled breath condensate accurate?}

\section{To the Editors:}

The metabolomic analysis of exhaled breath condensate (EBC) is a simple noninvasive approach for the study of respiratory system diseases. Previous studies introduced nuclear magnetic resonance (NMR)-based metabolomics as a method allowing a definite separation between healthy patients and patients with airway disease $[1,2]$. In these studies, the influence of external contaminants was also considered. DE LAURENTIIS et al. [1] reported that the removal of interfering residual external contaminants was crucial for correct EBC analysis and they proposed a cleaning protocol for the "complete removal of the disinfectant signals" from the reusable parts of the condensers.

In order to verify the influence of the disinfectant signals we have compared the EBC ${ }^{1} \mathrm{H}-\mathrm{NMR}$ spectra of a healthy subject obtained after the standard cleaning protocol (disinfected for 15 min using a $1.5 \%$ Descogen ${ }^{\mathrm{TM}}$ solution and flushed for 15 min with water) recommended by the manufacturer's guidelines and the International Consensus on EBC (fig. 1a) [3]; one that was obtained after the cleaning protocol proposed by DE LAURENTIIS et al. [1] (fig. 1b); and one obtained using a device without reusable condenser parts (fig. 1c). EBCs have been collected using an Anacon condenser (Biostec, Valencia, Spain). Sample collection and concentration ( $>99 \%$ water) were similar to those obtained in the procedure described by DE LAURENTIIS et al. [1] and a similar spectrum (fig. 1f) to the spectrum published by DE LAURENTIIS et al. [1] was obtained (fig. 1f, insert). Figure 1 shows that although many of the disinfectant signals present in the EBC spectrum have been removed by the DE LAURENTIIS et al. [1] cleaning protocol, there are some resistant peaks that are not observed in the spectrum in figure 1c. Furthermore, an EBC ${ }^{1} \mathrm{H}-\mathrm{NMR}$ spectrum of a healthy subject acquired using the device without reusable parts (fig. 1d) was compared with another control test (fig. 1e), collected in the same time scale using the condensation of room air pushed into the system by a continuous air spam device using flow and volumes similar to those typical of normal human respiratory function. In this case, it showed similar blank spectra with only two signals (lactate and an unidentified signal).

In our opinion, this comparison indicates that previous studies were not able to remove all disinfectant signals via the cleaning protocols. In addition, when the use of disinfectants was circumvented using a non-reusable device, we obtained blank spectra without metabolic information. We are persuaded to conclude that within the normal time periods used for EBC collection (10 min), NMR-based metabolomics does not have 